\title{
Calorimetric and Positron Lifetime Measurements of Hydrogenated Carbon Nanocones
}

\author{
A. BudziaK ${ }^{a, *}$, J. DRYZEK $^{a, b}$, J. KRAWCZYK $^{a}$ AND P.M. Zieliński ${ }^{a}$ \\ ${ }^{a}$ Institute of Nuclear Physics PAN, E. Radzikowskiego 152, 31-342 Kraków, Poland \\ ${ }^{b}$ Institute of Physics, University of Opole, Oleska 48, 45-052 Opole, Poland
}

\begin{abstract}
Two carbon nanostructured samples containing 5 and $20 \%$ of carbon nanocones in their volume were investigated. Using the Sieverts apparatus the hydrogen was loaded into the samples. The measurements of heat capacity in the temperature range from $100 \mathrm{~K}$ to $320 \mathrm{~K}$ and the positron lifetime measurements at the room temperature were performed for hydrogenated and non-hydrogenated carbon nanocones. The desorption of hydrogen at the temperature of $230 \mathrm{~K}$ is deduced from the heat capacity measurements. The detection of the positronium, the bound state of positron and electron, in the measured samples reveals the presence of open volume defects of $c a .0 .198 \pm 0.002 \mathrm{~nm}$.
\end{abstract}

PACS numbers: 81.05.U-, 78.70.Bj

\section{Introduction}

Efficient storage and delivery of hydrogen are key elements of the present economy. Hydrogen can be stored in various ways. The most popular are physical storage of compressed hydrogen gas in high pressure tanks, storage of liquid hydrogen in the insulated tanks and storage in advanced materials $[1,2]$. One of the promising materials comprise the carbon nanocones $(\mathrm{CNC})$, discovered in 1994 by Ge and Sattler [3]. CNC are the new form of carbon, fundamentally different from diamond, graphite and fullerenes/nanotubes. Preliminary patented [4] experimental results have demonstrated unprecedented $\mathrm{H}_{2}$ uptake-release unlike those for any other carbon material, as well as a new form of interaction between CNC and $\mathrm{H}_{2}$, in contrast to conventional physi- and chemisorption, capable of discharge at room temperature.

In our studies of CNC we performed the measurements of the heat capacity of CNC samples which were hydrogenated using the Sieverts apparatus. Additionally, the samples were investigated using the positron annihilation spectroscopy. This is a well established tool for investigation of the electronic properties and studies of open volume defects in condensed matter. It is due to the fact that the measured characteristics, i.e., the positron lifetime and the Doppler broadening of the annihilation line, reflect a local electron density in the spot where the annihilation takes place. Another important fact is that the presence of imperfections in the form of open volume defects in the atomic scale, e.g. vacancies, induces repulsion from positive ion cores. This causes positrons to get

* corresponding author; e-mail: andrzej.budziak@ifj.edu.pl trapped in these defects and an increase in its lifetime above the bulk value and the reduction of the Doppler broadening. In molecular solids in a large open volume or pores the positron and the electron create the bound state called the positronium (Ps), which is also easily recognized in the measured characteristics.

Positron annihilation characteristics were employed to the investigation of variety of materials including carbon. One can instance the value of positron lifetime: in diamond it is equal to $110 \mathrm{ps}$, in graphite (high oriented pyrolytic graphite, HOPG) to 212 ps and in fullerite sample, i.e. $\mathrm{C}_{60}-\mathrm{C}_{70}$ to $402 \mathrm{ps}$ [5]. In this paper we show the experimental values of the positron lifetime in powder samples of $\mathrm{CNC}$ both nonhydrogenated and hydrogenated.

\section{Experimental details}

\subsection{Hydrogen loading of carbon nanocones}

Carbon nanocones were produced in industrial quantities through the so-called Kvarner Carbon Black \& $\mathrm{H}_{2}$ $(\mathrm{CB} \& \mathrm{H})$ Process and composed of microstructures - flat nanodiscs and nanocones with five different apex angles - and soot [6].

Two carbon nanocones samples were used for the experiment, raw (CNC_05) and purified (CNC_20), containing ca. 5 and $20 \%$ of carbon nanocones in their volume, respectively. The flat discs and soot are the rest of the samples. The raw sample was obtained from n-TEC AS at Kjeller, Norway and then purified by University of Nottingham, England in the collaboration of the EU project HYCONES [7].

An UHV-compatible Sieverts apparatus to load the CNC with hydrogen was designed (Fig. 1). The exper- 
imental setup was used to measure adsorption and desorption isotherms volumetrically i.e. the change in pressure that occurs when the CNC are introduced into the volume containing the gas. The whole system is made of stainless steel (SS316). All elements were delivered by Svagelok. Before experiments the whole system was pumped out (below $10^{-8}$ mbar) and next baked out for $24 \mathrm{~h}$ at $200^{\circ} \mathrm{C}$. The sample itself was baked out for $12 \mathrm{~h}$ at $500{ }^{\circ} \mathrm{C}$. To keep the high accuracy of the experiments the setup was split into segments and volume of every one was estimated on the base of geometrical and $p-T-V$ measurements.

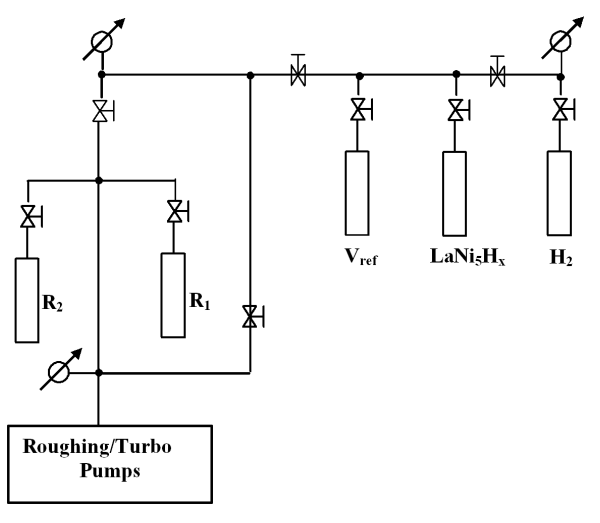

Fig. 1. Schematic view of the system used for measuring of the hydrogen uptake.

The differential hydrogen uptake was determined from the measured amount of hydrogen in the volume $R_{1}$ (sample holder), with and without CNC. Hydrogen (purity better than $99.99 \%$ ), accumulated in the hydrogen storage (based on $\mathrm{LaNi}_{5}$ ), was introduced into the volume $V_{\text {ref }}$, with the valve to $R_{1}$ closed, with pressure ranging from 0.1 to 7 bar. Thereafter a CNC sample of known weight was exposed to hydrogen by opening the valve to $R_{1}$. When pressure equilibrium was reached, the sample holder with the sample was cooled to $77 \mathrm{~K}$. After reaching a new pressure equilibrium the sample was exposed to a new dose of hydrogen — this process was repeated cumulatively up to a maximum pressure of $\approx 7$ bar. This means the errors of the measurements in every step were cumulated as well. Next, the valve to $R_{1}$ was closed, volume $V_{\text {ref }}$ pumped out and the sample holder $R_{1}$ was warmed above the room temperature and hydrogen desorbed from the sample was accumu-

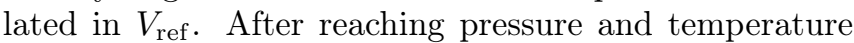
equilibrium it was possible to calculate the total amount of hydrogen accumulated by the sample. The samples CNC_05 and CNC_20 after hydrogenating are called $\mathrm{CNC} \overline{\mathrm{H}} \_05$ and $\mathrm{CNCH} \overline{C H}_{-} 20$ in the following text.

\subsection{Heat capacity measurements}

Calorimeter cell having an in-built heater and a thermometer was filled with the sample, vacuum-tight sealed, positioned inside two concentric shields each having its temperature stabilized, and finally closed inside a steel container. The system was evacuated down to $c a$. $10^{-6}$ mbar and put inside a liquid nitrogen cryostat.

During the measurement constant portions of energy were periodically introduced to the sample and resulting changes of its temperature were recorded as a function of time. The temperature of the sample was measured by a platinum resistance thermometer with a sensitivity of $\approx 10 \mu \mathrm{K}$. The accuracy of the specific heat measured in adiabatic conditions was better than $1 \%$. On that basis the heat capacity as a function of temperature was calculated for $\mathrm{CNC}_{2} 20$ and $\mathrm{CNCH}_{-} 20$ samples.

\subsection{Positron lifetime measurements}

For the positron lifetime measurement the ${ }^{22} \mathrm{NaCl}$ positron emitter enveloped in the $7 \mu \mathrm{m}$ kapton foil was embedded in or sandwiched between the powder sample of the CNC with such a quantity that ensured positron annihilation in the sample. The sample and the positron source were encapsulated in the glass test tube of diameter $15 \mathrm{~mm}$ which was pumped out and sealed. The test tube was located in front of the detectors of the positron lifetime spectrometer. The positron lifetime spectra were measured using the conventional fast-fast spectrometer with $\mathrm{BaF}_{2}$ scintillators. The time resolution of the system was 250 ps (FWHM). All obtained spectra of about $2 \times 10^{6}$ counts were deconvoluted subtracting the background and the source component.

\section{Results and discussion}

The density of the investigated samples was estimated to be $1.8 \pm 0.4 \mathrm{~g} / \mathrm{cm}^{3}$, which corresponds to density of graphite $\left(2.0-2.2 \mathrm{~g} / \mathrm{cm}^{3}\right)$. The hydrogen uptake as a function of applied hydrogen pressure for CNC_05 and CNC 20 samples obtained at $77 \mathrm{~K}$ is presented in Fig. 2. At hydrogen pressure of 7 bar maximum differential uptake was determined to be 1.1 weight percent (wt\%) for CNC 05 and $4.0 \mathrm{wt} \%$ for CNC 20, respectively. Because the content of carbon nanocones in CNC_20 is also four times higher than in CNC_05, it can indicate that the carbon nanocones are mainly responsible for the uptake of hydrogen in both samples. The participation of carbon nanodiscs and soot in the process of hydrogenation seems to be minor. At room temperature and hydrogen pressure of 7 bar the maximum uptake was a little above $0.2 \mathrm{wt} \%$ for CNC 20 and $c a$. $0.1 \mathrm{wt} \%$ for CNC 05 .

It has been shown [8] that in case of carbon nanotubes specific heat is mainly determined by contribution from phonons and depends on nanotube radius and temperature in such a way that for small values of tube's radius and low temperatures $C_{\mathrm{ph}} \sim T$, otherwise $C_{\mathrm{ph}} \sim T^{2}$. Since $\mathrm{CNC}$ are made of graphene sheets just like in case of carbon nanotubes and our sample consists of 2-dimensional carbon cones, discs and graphite planes, we fitted a second-order polynomial to the experimental data. A good agreement between proposed curve and 


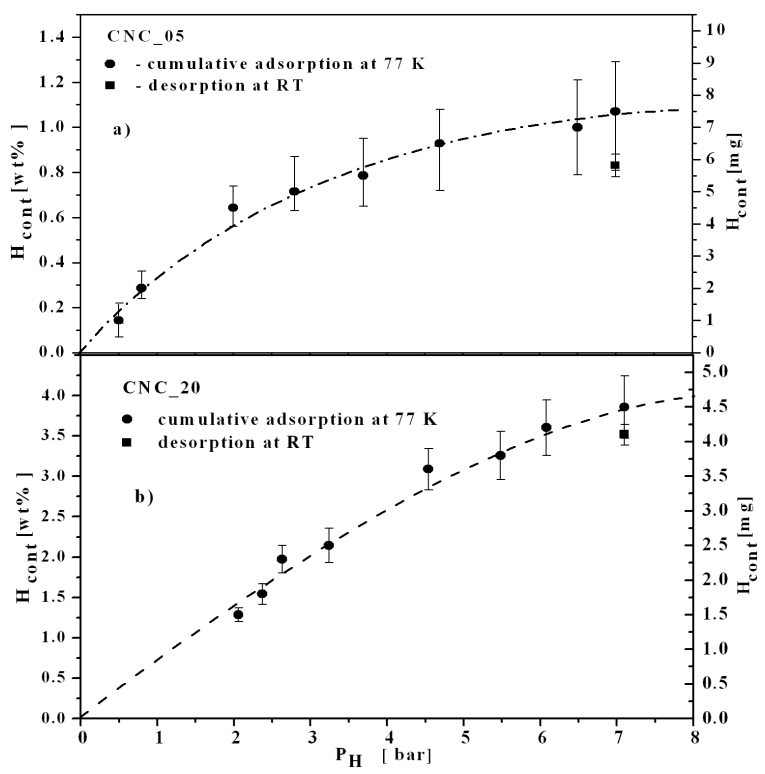

Fig. 2. The hydrogen uptake as a function of applied hydrogen pressure for CNC_05 (a) and CNC_20 (b) samples at $77 \mathrm{~K}$.

measured data was found (Fig. 3). Significant increase in heat capacity of the hydrogenated sample as compared to the sample without hydrogen is clearly visible between $100 \mathrm{~K}$ and $230 \mathrm{~K}$. Above $230 \mathrm{~K}$ and up to $320 \mathrm{~K}$ heat capacity of $\mathrm{CNC} \_20$ and $\mathrm{CNCH} 20$ samples is almost the same. This might suggest that around $230 \mathrm{~K}$ desorption of hydrogen from CNC occurred. On the other hand, no evidence of such process around $150 \mathrm{~K}$ and $300 \mathrm{~K}$ was observed and that was to be expected taking into account hydrogen desorption curve for CNC [4].

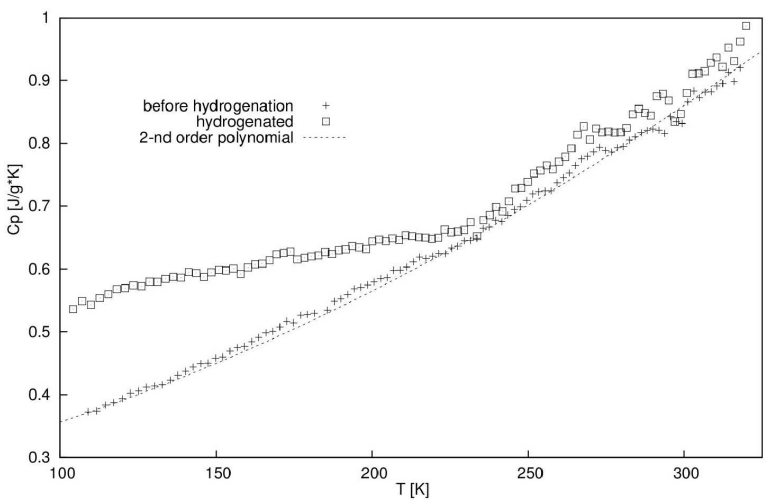

Fig. 3. The temperature dependence of the heat capacity measured for non-hydrogenated and hydrogenated CNC_20 samples.

Unfortunately, the amount of the $\mathrm{CNC}$ in the sample is of order of $20 \%$, therefore obtained results correspond to contribution from 2-dimensional carbon discs and graphite sheets as well and therefore should be treated as preliminary and at most qualitative.
Two measurements of the positron lifetime spectrum were performed on the non-hydrogenated (CNC_05) and hydrogenated $(\mathrm{CNCH}$ 05) samples. The measured spectra are shown in Fig. 4 and significant difference between them is clearly visible. The deconvolution of the spectra reveals two lifetime components in each spectrum. For non-hydrogenated sample the lifetime of the first component was equal to $387 \pm 2$ ps and of the second component to $1214 \pm 15$ ps. The intensities of the first and second components were $84.3 \pm 0.3 \%$ and $15.7 \pm 0.3 \%$, respectively. After loading of the hydrogen, the value of the first lifetime component was slightly reduced to the value of $374 \pm 2 \mathrm{ps}$, but its intensity increased to $96.2 \pm 0.3 \%$. The significant change was observed in the second lifetime component (Fig. 4). The value of lifetime was reduced to $947 \pm 22$ ps and the intensity decreased almost four times to the value of $3.8 \pm 0.3 \%$.

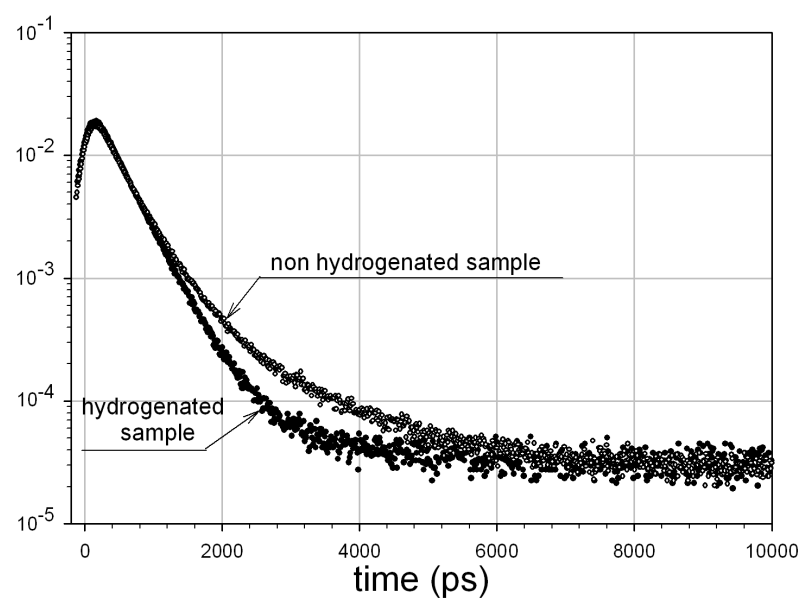

Fig. 4. Positron annihilation lifetime spectra measured at the room temperature for non-hydrogenated (open circles) and hydrogenated (close circles) CNC_05 samples.

The interpretation of the obtained results is as follows. The value of the first lifetime component corresponds well with those reported by other authors in fine grained nuclear graphite samples, i.e. 362 ps (AXF-5Q1), 369.2 ps (IG-110) and glass carbon (GC-10) 370.2 ps [9]. One should mention that positron lifetime for pristine multi-walled carbon nanotubes and cup-stacked carbon nanotubes is in the range of $360 \mathrm{ps}$ [10]. It is commonly accepted that such a value results from positrons annihilating in the open volume defect clusters like pores. Annihilation in such case occurs at the internal surface of the pores and the values obtained in our case are closely in agreement with those reported by others as well in graphite [11, 12]. Many authors in general have attributed lifetimes ranging from 140 to 225 ps to free positron annihilation with $\pi$-electrons in the whole volume of the graphite. Lack of such value in our spectra is caused by the large concentration of such defects which saturates positrons trapping. Slight reduction of 
the value of this lifetime component after hydrogen loading does not change this interpretation.

The most surprising result is the presence of the second lifetime component in the measured spectra. Its value above or close to $1 \mathrm{~ns}$ points out to the presence of large pores, where the Ps is formed. Taking into account the Tao-Eldrup model we can estimate the radius of the pore to be equal to $0.198 \pm 0.002 \mathrm{~nm}$ for the case of the non-hydrogenated sample and to $0.156 \pm 0.004 \mathrm{~nm}$ for the hydrogenated sample. Another surprising result is that the intensity of this component decreases after the hydrogen loading $c a$. four times. Owing to the fact that the Ps and hydrogen have identical diameters, both particles can occupy the same pores. The presence of hydrogen in the pore after the loading of the sample reduces the number of the pores where Ps could be formed and finally reduces the intensity of the lifetime component attributed to the Ps formation and annihilation. It seems that the bounding of hydrogen in such a pore takes place. Determination of the binding energy between hydrogen and a pore needs future studies. Hydrogen trapping in vacancies was experimentally observed in copper [13]. Thus the binding of hydrogen with such pores and not the other defects present in nanocones seems to be the most surprising result detected by positron annihilation in these studies.

\section{Conclusions}

We have measured adsorption isotherms by the Sieverts method, heat capacity and positron lifetime in the nanostructured carbon samples containing 5 and $20 \%$ of carbon nanocones.

The maximum total hydrogen uptake at $77 \mathrm{~K}$ and 7 bar hydrogen pressure was determined to be roughly 1.1 and $4.0 \mathrm{wt} \%$ for CNC 05 and $\mathrm{CNC}$ 20, respectively. At room temperature the uptake is much smaller, which is expected for non-activated adsorption. The results show that the raw, non purified CNC 05 sample is a very poor material as a potential hydrogen storage material. However, the results for the CNC 20 are comparable to previous experimental works on different carbon nanostructures such as single and multiwalled nanotubes, nanofibers and activated carbon [12-16], but are still far from required by US Department of Energy value of $6.5 \mathrm{wt} \%$ percent in storage material at ambient temperature and pressure.

The preliminary measurements of the heat capacity of the CNC samples reveal in the temperature range between $100 \mathrm{~K}$ and $230 \mathrm{~K}$ the increase in heat capacity of the hydrogenated sample, as compared to the sample without hydrogen. Nevertheless, above the temperature of $230 \mathrm{~K}$ the heat capacity of both samples is almost the same, which suggests the desorption of hydrogen at this temperature. The positron lifetime measurements show that in hydrogenated and non-hydrogenated CNC 05 sample the creation of Ps takes place, which indicates the presence of the open volume defects of size of $c a$. $0.156 \pm 0.004 \mathrm{~nm}$ and $0.198 \pm 0.002 \mathrm{~nm}$, respectively. We observed also the reduction in the intensity of Ps formation in the hydrogenated sample, which suggests that the hydrogen is likely to be loaded into the open volume defect.

The results presented in this paper show that carbon nanocones can be promising material for hydrogen storage, although further purification of the samples is essential in order to perform more in-depth research of the nature of CNC-hydrogen binding mechanism. Another interesting project would be to study physical properties of the CNC decorated with various metals such as platinum and palladium.

\section{Acknowledgments}

The funding by the European Commission (Project No. NMP3-CT-2006-032970/HYCONES) is gratefully acknowledged.

\section{References}

[1] Y. Zhao, Y.-H. Kim, A.C. Dillon, M.J. Heben, S.B. Zhang, Phys. Rev. Lett. 94, 155504 (2005).

[2] A. Zuttel, Naturwiss. 91, 157 (2004).

[3] M. Ge, K. Sattler, Chem. Phys. Lett. 220, 192 (1994).

[4] US Patent No. 6, 290, 753 B1, issued Sept. 18, 2001 to A.T. Skjeltorp and A. Maeland.

[5] Y. Ito, T. Suzuki, Phys. Rev. B 60, 15636 (1999).

[6] A. Krishnan, E. Dujardin, M.M.J. Treacy, J. Hugdahl, S. Lynum, T.W. Ebbesen, Nature 388, 451 (1997).

[7] Hydrogen Storage in Carbon Cones HYCONES, STREP NMP3-CT-2006-032970, http://www . hycones.eu/ .

[8] L.X. Benedict, S.G. Louie, M.L. Cohen, Solid-State Commun. 100, 177 (1996).

[9] M. Hasegawa, M. Kajino, H. Kuwahara, E. Kuramoto, M. Takenaka, S. Yamaguchi, Mater. Sci. Forum 105-110, 1041 (1992).

[10] H. Murakamia, K. Sato, I. Kanazawa, M. Sano, Acta Phys. Pol. A 113, 1479 (2008).

[11] K. Chakrabarti, P.M.G. Nambissan, C.D. Mukherjee, K.K. Bardhan, C. Kim, K.S. Yang, Carbon 44, 948 (2006).

[12] J. Dryzek, E. Pamula, S. Błażewicz, Phys. Status Solidi A 151, 39 (1995).

[13] B. Lengeler, S. Mantl, W. Triftshäuser, J. Phys. F, Met. Phys. 8, 1691 (1978).

[14] A.C. Dillon, K.M. Jones, T.A. Bekkedahl, C.H. Kiang, D.S. Bethune, M.J. Heben, Nature 386, 377 (1997).

[15] Q.Y. Wang, J.K. Johnson, J. Phys. Chem. B 103, 4809 (1999).

[16] N. Texier-Mandoki, J. Dentzer, T. Piquero, S. Saadallah, D.P. Vix-Guterl, Carbon 42, 2735 (2004). 